\title{
Diurnal Temperature Profile Impacts on Estimating Effective Soil Temperature at L-Band
}

\author{
P.M. O'Neill $^{\mathrm{a}}{ }^{\mathrm{b} *}$, R. Dehaan ${ }^{\mathrm{a} c}$ and J.P. Walker ${ }^{\mathrm{d}}$ \\ ${ }^{a}$ E.H. Graham Centre for Agricultural Innovation (NSW Department of Primary Industries and Charles \\ Sturt University), Charles Sturt University, Wagga Wagga, New South Wales, 2678 \\ ${ }^{\mathrm{b}}$ School of Computing \& Mathematics, Charles Sturt University, Wagga Wagga, New South Wales, 2678 \\ ${ }^{c}$ School of Environmental Sciences, Charles Sturt University, Wagga Wagga, New South Wales, 2678 \\ ${ }^{\mathrm{d}}$ Department of Civil Engineering, Monash University, Clayton, Victoria, 3800 \\ Email:Paul.ONeill@aip.org.au
}

\begin{abstract}
Near-surface soil moisture is an important parameter in hydrological, meteorological and agricultural applications. Passive microwave observations, both airborne and satellite, can be used to infer near-surface soil moisture. The brightness temperature of the thermal radiation that crosses the soil-air interface is the product of the soil emissivity and the effective temperature. At L-band, the emissivity is sensitive to the moisture content in the top few to several centimetres, while the effective temperature is the weighted average of the emission from all locations within the soil. The effective temperature is used to normalise the observed brightness temperature so that the near-surface soil moisture can be inferred.
\end{abstract}

The effective temperature is a function of the soil temperature and moisture profiles, full information of which is not available for remote sensing applications. The so-called 'C-parameterisation' uses two temperatures, one at or near the soil surface and the other at depth, to estimate the effective temperature. Variations in the shape of the temperature profile are not taken into account, so diurnal temperature variations might impact on the effectiveness of this method. Moreover, the best results have been obtained when the temperature just below the surface $($ e.g., $5 \mathrm{~cm}$ ) is used. Operational applications thus rely on simulations to provide the necessary sub-surface temperature information.

The primary aim of the work presented here is to investigate the influence of diurnal temperature variations on the effectiveness of the C-parameterisation. A new method (the 'ratio model') is then introduced by which $T_{\mathrm{EFF}}$ may be estimated solely from thermal infrared measurements of the soil skin temperature. The Simultaneous Heat and Water model of Flerchinger et al. (1998) was used to simulate temperature and moisture profiles under bare soil. The daytime profiles were used, with the aim of developing a model for use primarily with airborne observations acquired throughout the day.

The C-parameterisation performed best when the temperature at a depth of $5 \mathrm{~cm}$ was used, with an RMS error of $0.29 \mathrm{~K}$. When the temperature at the soil surface was used instead, the RMS error increased to $1.7 \mathrm{~K}$ and the residuals exhibited an increase from -2 to $3 \mathrm{~K}$ between 10:00 and 18:00 hours. This trend is owing to the phase difference between the effective temperature and soil skin temperature. The skin temperature peaks at $\sim$ 13:00 hours, while the effective temperature peaks $\sim 3$ hours later.

The ratio between the effective temperature and soil skin temperature was found to vary smoothly, decreasing to a minimum near the middle of the day. By fitting a 3-parameter model to the data, the effective temperature could be estimated as a function of the skin temperature and hour of day. With an RMS error of $0.95 \mathrm{~K}$, this model outperformed the C-parameterisation when using the soil skin temperature. The ratio model is primarily expected to be beneficial for airborne operations. A modified form may be required to account for variations in latitude, season, soil and vegetation properties, and meteorological conditions.

Keywords: Microwave radiometry, remote sensing, soil measurements, temperature

\footnotetext{
*Present address: Fugro Seismic Imaging Pty Ltd, 69 Outram Street, West Perth, Western Australia, 6005
} 


\section{INTRODUCTION}

Near-surface soil moisture is an important parameter in hydrological, meteorological and agricultural applications. In agriculture, the near-surface soil moisture affects seed germination and the breakdown of organic material. Moreover, measurements of the near-surface moisture can be assimilated into models to predict the root-zone soil moisture (Das et al., 2008).

Passive microwave observations, both airborne and satellite, can be used to infer near-surface soil moisture (Ulaby et al., 1986). Thermal radiation is emitted from every location within the soil and is attenuated as it propagates to the surface. In the zero-order noncoherent model, the brightness temperature of the radiation that crosses the soil-air interface is the product of the soil emissivity and the effective soil temperature, $T_{\mathrm{EFF}}$. At L-band, the emissivity is sensitive to the moisture content in the top few to several centimetres. The effective temperature is the weighted average of the thermodynamic temperatures at all depths and is a function of both the temperature and moisture profiles, the latter of which determines the temperature sensing depth. $T_{\mathrm{EFF}}$ is required to normalise the observed microwave brightness temperature so that the emissivity can be determined and the soil moisture inferred.

Full information on the temperature and moisture profiles is not available for remote sensing applications, so methods have been developed to estimate $T_{\mathrm{EFF}}$ using the soil temperature at certain depths (e.g., Choudhury et al., 1982; Wigneron et al., 2001, 2008; de Rosnay et al., 2006). In the so-called 'Cparameterisation' two temperatures are used: one either at or near the soil surface and the other at depth. The near-surface soil water content can also be incorporated to account for moisture-dependent variations in the temperature sensing depth (Wigneron et al., 2001).

There are two potential drawbacks associated with using the C-parameterisation. First, variations in the shape of the temperature profile are not taken into account. Diurnal variations might thus impact on the effectiveness of this method. Second, operational applications rely on simulations to provide the necessary sub-surface temperature information. The soil skin temperature can be measured using thermal infrared observations. However, the best results using the C-parameterisation have thus far been obtained when the temperature just below (e.g., $5 \mathrm{~cm}$ ) the surface is used (e.g., Wigneron et al., 2008).

The primary aim of the work presented here is to investigate the influence of diurnal temperature variations on the effectiveness of the C-parameterisation. A new method (the 'ratio model') is then introduced by which $T_{\mathrm{EFF}}$ may be estimated solely from thermal infrared measurements of the soil skin temperature.

The relationship between the effective and thermodynamic temperatures has been studied previously using temperature and moisture profiles observed during a dry down period (Choudhury et al., 1982). Wigneron et al. (2008) used simulations of a dry down to investigate the influence of soil properties on effective temperature. Following the same approach, the present work uses profiles generated by a mechanistic heat and water transfer model under bare soil. The daytime profiles are considered, with the aim of developing a model that would especially benefit moisture retrievals from airborne observations acquired throughout the day.

\section{SOIL HEAT AND WATER SIMULATIONS}

The Simultaneous Heat And Water (SHAW) model was used to generate synthetic soil temperature and moisture profiles (for a description and validation see Flerchinger et al., 1998, and references therein). SHAW simulates the flow of heat, water and solutes though a one-dimensional soil-vegetation-atmosphere system. Up to fifty soil nodes, with corresponding properties (e.g., texture, hydraulic properties), can be specified.

A bare soil simulation was conducted for a silty clay loam soil (11\% sand, $27 \%$ clay) with the following properties: bulk density $1420 \mathrm{~kg} \mathrm{~m}^{-3}$, saturated volumetric water content $0.44 \mathrm{~cm}^{3} \mathrm{~cm}^{-3}$, pore size distribution index 5.5 , air-entry potential $-0.2 \mathrm{~m}$, saturated conductivity $0.439 \mathrm{~cm} \mathrm{~h}^{-1}$, dry soil albedo 0.2 , soil albedo exponent 1.0. The profile was modelled with 26 nodes, defined to a depth of $4 \mathrm{~m}$, with 11 nodes being used in the top $5 \mathrm{~cm}$. The simulation had a duration of 40 days, with 1 hour time steps. The first 10 days were used to initialise the model. A constant precipitation rate of $25 \mathrm{~mm} \mathrm{~h}^{-1}$ simulated during this time saturated the soil profile. The last 30 days then comprised a dry down period, during which there were no wetting events. A single 24 hour set of forcing data was used for all 30 days, rep- 
resenting typical, clear sky summer conditions in the Riverina region of New South Wales. The daytime (07:00-18:00 hours) profiles from these last 30 days are used in this study. The $0-3 \mathrm{~cm}$ volumetric water content varied in the range $0.15-0.38 \mathrm{~cm}^{3} \mathrm{~cm}^{-3}$.

The theoretical effective temperature of each synthetic profile was calculated using

$$
T_{\mathrm{EFF}}=\int_{0}^{\infty} T(z) \alpha(z) \exp \left[-\int_{o}^{z} \alpha\left(z^{\prime}\right) d z^{\prime}\right] d z
$$

where $T$ and $\alpha$ are, respectively, the soil thermodynamic temperature and attenuation coefficient at depth $z$ (Choudhury et al., 1982). The attenuation coefficient is a function of the real, $\varepsilon^{\prime}$, and imaginary, $\varepsilon^{\prime \prime}$, components of the dielectric constant, and is expressed as

$$
\alpha(z)=\frac{4 \pi}{\lambda} \varepsilon^{\prime \prime}(z) / 2\left(\varepsilon^{\prime}(z)\right)^{1 / 2}
$$

where $\lambda$ is the microwave emission wavelength. The model of Wang \& Schmugge (1980) was used to relate $\varepsilon^{\prime}$ and $\varepsilon^{\prime \prime}$ to the volumetric water content, at a microwave frequency of $1.4 \mathrm{GHz}$.

\section{ESTIMATING $T_{\mathrm{EFF}}$ USING THE C-PARAMETERISATION}

Prior to introducing a new method of estimating $T_{\mathrm{EFF}}$, the effectiveness of the C-parameterisation was assessed. The influence of diurnal variations on estimating $T_{\mathrm{EFF}}$ using this method is demonstrated.

\subsection{Model formulation}

In the C-parameterisation, the effective temperature is modelled as a function of the soil near surface temperature, $T_{\text {surf }}$, deep soil temperature, $T_{\infty}$, and a coefficient $C$ using (Choudhury et al., 1982)

$$
T_{\mathrm{EFF}}=T_{\infty}+\left(T_{\mathrm{surf}}-T_{\infty}\right) C
$$

The range of soil depths that contribute to $T_{\mathrm{EFF}}$ is smaller for a wet soil than for a dry soil. For a given temperature profile, $C$ is therefore expected to increase with soil moisture. Wigneron et al. (2001) accounted for the moisture dependence by expressing the coefficient $C$ as a function of the $0-3 \mathrm{~cm}$ volumetric water content, $w_{\mathrm{S}}$. The depth of the $0-3 \mathrm{~cm}$ layer corresponds well to the L-band moisture sensing depth. The Wigneron et al. (2001) formulation has the form

$$
T_{\mathrm{EFF}}=T_{\infty}+\left(T_{\mathrm{surf}}-T_{\infty}\right)\left(\frac{w_{\mathrm{S}}}{w_{0}}\right)^{b}
$$

where the parameters $w_{0}$ and $b$ allow the model to be calibrated. The temperatures at various depths have been used for $T_{\text {surf }}$ and $T_{\infty}$. The best estimates of $T_{\mathrm{EFF}}$ have been obtained when using the temperature at a few to several centimetres for $T_{\text {surf }}$, and with the temperature at $50 \mathrm{~cm}$ being used for $T_{\infty}$ (Wigneron et al., 2001; de Rosnay et al., 2006; Wigneron et al., 2008).

\subsection{Modelling results}

The synthetic temperature and moisture profiles were used to assess the effectiveness of the Cparameterisation. The temperatures at depths of $5 \mathrm{~cm}\left(T_{5}\right)$ and $50 \mathrm{~cm}\left(T_{50}\right)$ were used, along with the soil skin temperature (i.e., the temperature of the node at $0 \mathrm{~cm}, T_{0}$ ). The mean $0-3 \mathrm{~cm}$ volumetric water content was computed for each moisture profile. The formulation given in (4) was used to perform two tests. The shallow temperature was defined to be $T_{\text {surf }}=T_{5}$ in the first test, and then $T_{\text {surf }}=T_{0}$ in the second. The deep soil temperature was defined to be $T_{\infty}=T_{50}$ in both cases. 
Table 1. Root-mean-square error and bias associated with estimating $T_{\mathrm{EFF}}$ using the C-parameterisation and the ratio model. A positive bias corresponds to the model underestimating $T_{\mathrm{EFF}}$.

\begin{tabular}{lcc}
\hline Model $\left(T_{\text {surf }}\right)$ & \multicolumn{2}{c}{$T_{\mathrm{EFF}}$} \\
& $\begin{array}{c}\text { RMS error } \\
(\mathrm{K})\end{array}$ & $\begin{array}{c}\text { Bias } \\
(\mathrm{K})\end{array}$ \\
\hline $\mathrm{C}\left(T_{5}\right)$ & 0.29 & 0.058 \\
$\mathrm{C}\left(T_{0}\right)$ & 1.7 & -0.22 \\
ratio & 0.95 & 0.016 \\
\hline
\end{tabular}

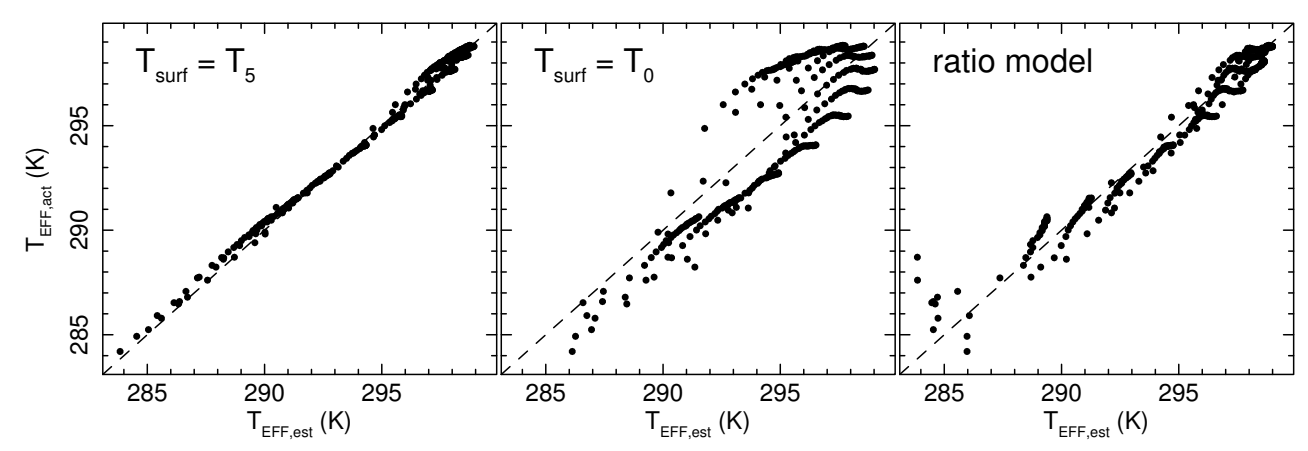

Figure 1. Actual effective temperature $\left(T_{\mathrm{EFF}, \text { act }}\right)$ versus estimated effective temperature $\left(T_{\mathrm{EFF}, \mathrm{est}}\right)$ determined using the C-parameterisation (left two panels) and ratio model (right-hand panel). The left-hand and middle panels, respectively, show estimates using $T_{\text {surf }}=T_{5}$ and $T_{\text {surf }}=T_{0}$.

The parameters $w_{0}$ and $b$ were varied so as to minimise the RMS error between the actual effective temperature calculated with (1), $T_{\mathrm{EFF}, \text { act }}$, and that estimated by the C-parameterisation using (4), $T_{\mathrm{EFF}}$,est. For the case of $T_{\text {surf }}=T_{5}$, the best-fitting values of $w_{0}$ and $b$ were $0.653 \mathrm{~cm}^{3} \mathrm{~cm}^{-3}$ and 0.287 , respectively. For $T_{\text {surf }}=T_{0}$ these values were $1.81 \mathrm{~cm}^{3} \mathrm{~cm}^{-3}$ and 0.426 .

The RMS error and bias for both models are presented in Table 1, and the relationship between $T_{\mathrm{EFF} \text {,est }}$ and $T_{\mathrm{EFF} \text {, act }}$ is shown in Fig. 1. The residuals associated with estimating $T_{\mathrm{EFF}}$ are presented in Fig. 2. As expected, (4) performs very well for the case of $T_{\text {surf }}=T_{5}$ (e.g., Wigneron et al., 2008). However, when the skin temperature is used the effectiveness of the C-parameterisation is reduced. The residuals in this case exhibit a systematic increase from approximately -2 to $3 \mathrm{~K}$ between 10:00 and 18:00 hours.

The value of $C$ for each profile that would yield $T_{\mathrm{EFF} \text {, est }}=T_{\mathrm{EFF} \text {, act }}$ was calculated, and the mean value of $C$ was determined as a function of hour. Fig. 3 shows the mean values of $C$ during the first and second halves of the dry down period, as well as those for all 30 days. The corresponding mean values of $w_{\mathrm{S}}$ are also plotted. The diurnal variations of the soil temperature can be seen in Fig. 3, where the mean temperature at various depths, and the effective temperature, are plotted as a function of hour.

Between 07:00 and 09:00 hours, $T_{\text {surf }}$ is increasing most rapidly and the temperature difference between $T_{\text {surf }}$ and $T_{\infty}$ is minimised. Indeed, at 08:00 hours, the absolute difference between $T_{0}$ and $T_{50}$ was only $0.1 \mathrm{~K}$ on the first day of the dry down period and $0 \mathrm{~K}$ on the second day (these two outliers were excluded from the calculation of the mean values of $C$ ). The value of $C$ is therefore unstable in the early morning, and during this time the estimated effective temperature is least sensitive to the value of $C$. Negative values of $C$ occur in the early morning when the soil surface has been heated sufficiently to raise $T_{0}$ above $T_{50}$, prior to the deeper layers also being heated enough to raise $T_{\mathrm{EFF}}$ above $T_{50}$.

The value of $C$ is expected to decrease during the afternoon as the soil dries and the temperature sensing depth increases. However, for the case of $T_{\text {surf }}=T_{0}, C$ actually increased from 0.18 to 0.68 between 


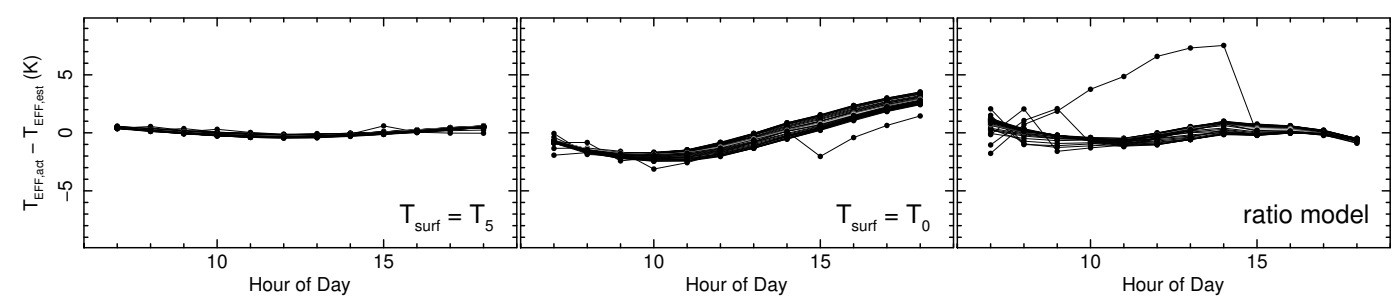

Figure 2. Residuals associated with estimating the effective temperature using the C-parameterisation (left-hand and middle panels) and the ratio model (right-hand panel), plotted as a function of hour of day. The left-hand and middle panels correspond to estimates using $T_{\text {surf }}=T_{5}$ and $T_{\text {surf }}=T_{0}$, respectively. The solid lines join points from the same day. A positive error corresponds to an underestimate of $T_{\mathrm{EFF}}$. The line peaking at a residual temperature of $7.5 \mathrm{~K}$ in the right-hand panel corresponds to the first day of the dry down period.

10:00 and 18:00 hours. As seen in Fig. 2, the residuals exhibit a corresponding trend. This variation can be attributed to a phase difference between $T_{0}$ and $T_{\mathrm{EFF}} . T_{0}$ peaks at $\sim 13: 00$ hours while $T_{\mathrm{EFF}}$ continues to rise until $\sim 16: 00$ hours. $T_{5}$ and $T_{\mathrm{EFF}}$ peak within 1 hour of each other, so the increase in $C$ is not as large for the case of $T_{\text {surf }}=T_{5}$. Note that the diurnal variations in the temperature profile do not entirely obscure the expected dependence of $C$ on soil moisture: $C$ is higher during days $1-15$ of the dry down period than it is during days 16-30.

\section{ESTIMATING $T_{\mathrm{EFF}}$ USING THE RATIO MODEL}

Here, a method is developed by which the effective temperature may be estimated from thermal infrared measurements of the soil skin temperature only. The ratio between $T_{\mathrm{EFF}}$ and $T_{0}$, denoted as $\rho$, was calculated for each synthetic profile. Fig. 3 shows the variation in the mean value of $\rho$ with hour of day. The diurnal variations are smooth, with $\rho$ decreasing to a minimum at approximately midday. The method described below seeks to estimate $\rho$ for bare soil and, when combined with a measurement of the skin temperature, yield an estimate of $T_{\mathrm{EFF}}$.

\subsection{Model formulation}

The effective soil temperature and the temperature at the soil surface can be related by the following expression

$$
T_{\mathrm{EFF}}=\rho T_{0}
$$

The dependence of $\rho$ on hour is described by the expression

$$
\rho=1-\left(1-\rho_{\min }\right) \sin \left(\frac{\pi}{2 P}\left[H-H_{0}\right]\right)
$$

where $H$ is the hour of day. The parameter $\rho_{\min }$ is the minimum value of $\rho$, which occurs at close to midday, and $H_{0}$ is the hour at which $\rho$ equals unity, which occurs early in the morning (see Fig. 3 ). The parameter $P$ is related to the period of variation in $\rho$, and is equal to the time difference between $H_{0}$ and that at which $\rho=\rho_{\min }$.

\subsection{Modelling results}

The parameters $\rho_{\min }, H_{0}$ and $P$ were varied so as to minimise the RMS error between $T_{\mathrm{EFF} \text {, act }}$ and $T_{\mathrm{EFF}, \text { est }}$. The best-fitting values of $\rho_{\mathrm{min}}, H_{0}$ and $P$ were $0.961,7.22$ hours and 5.76 hours, respectively. 

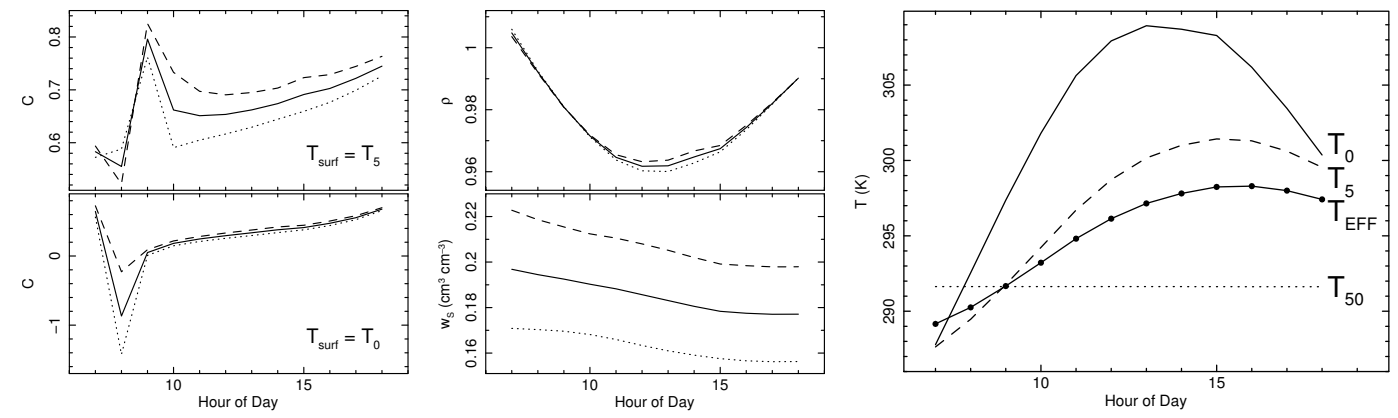

Figure 3. Mean values of $C$ (left-hand panels), ratio between effective temperature and soil skin temperature ( $\rho$; middle panel, top), $0-3 \mathrm{~cm}$ volumetric water content ( $w_{\mathrm{S}}$, middle panel, bottom), and soil temperature (right-hand panel) as a function of hour of day. In the left-hand and middle panels the solid lines show the means for the entire dry down period. The dashed and dotted lines in those plots correspond to days 1-15 and 16-30, respectively. The left-hand pair of panels show $C$ calculated using $T_{\text {surf }}=T_{5}$ and $T_{\text {surf }}=T_{0}$, as indicated. In the right-hand panel the mean effective temperature is shown as the solid line with markers. $T_{0}, T_{5}$ and $T_{50}$ are show as solid, dashed and dotted lines, respectively.

The RMS error and bias are given in Table 1, and the relationship between $T_{\mathrm{EFF} \text {,est }}$ and $T_{\mathrm{EFF} \text {,act }}$ is shown in Fig. 1. The residuals are presented in Fig. 2. The model performed worst during the first five days of the dry down, when $w_{\mathrm{S}}$ was highest, and distinct variations can be seen in the residuals. Overall, however, the ratio model performed well. It outperformed the C-parameterisation using $T_{\text {surf }}=T_{0}$, but was not as effective as that using $T_{\text {surf }}=T_{5}$.

Note that, in Fig. 3, the mean value of $\rho$ at each hour between 09:00 and 17:00 hours decreased during the dry down period. The day-to-day solar forcing was constant throughout the simulation, so this reduction in the mean $\rho$ can be related to the reduction in soil moisture during the dry down.

\section{DISCUSSION AND CONCLUSIONS}

Determining the effective soil temperature is a crucial aspect of retrieving near-surface soil moisture from passive microwave observations. In the present study, simulated temperature and moisture profiles, corresponding to a 30 day dry down period, have been used to investigate the influence of diurnal temperature variations on estimating $T_{\mathrm{EFF}}$. The popular C-parameterisation has been tested alongside a new method (the ratio model) based on the ratio between the soil effective temperature and skin temperature.

As expected from previous studies, (e.g., Wigneron et al., 2008), the C-parameterisation performed better for the case of $T_{\text {surf }}=T_{5}$ than for $T_{\text {surf }}=T_{0}$. In the former case, the residuals exhibited a diurnal variation of only $\sim 1 \mathrm{~K}$, while in the latter the residuals increased by $\sim 5 \mathrm{~K}$ during the afternoon. This trend is owing to the time lag between the variations in $T_{0}$ and $T_{\mathrm{EFF}}$, with the temperature at the soil surface peaking 3 hours prior to the effective temperature. In the formulation of the $\mathrm{C}$-parameterisation, the value of $C$ is assumed to be independent of the shape of the temperature profile. For constant values of $T_{\infty}$ and $w_{\mathrm{S}}$, the effective temperature estimated by this method will thus exhibit variations in phase with those of $T_{\text {surf }}$. Given that the effective temperature is a weighted average of the sub-surface soil temperatures, it is not surprising that the variations in $T_{\mathrm{EFF}}$ could be better represented by $T_{5}$.

The ratio, $\rho$, between $T_{\mathrm{EFF}}$ and the soil skin temperature was found to vary smoothly between 07:00 and 18:00 hours, reaching a minimum near midday. The ratio model was formulated to estimate $T_{\mathrm{EFF}}$ from $\rho$ and the skin temperature, with $\rho$ being modelled as a sinusoidal function of hour of day. This method was not as effective as the C-parameterisation using $T_{\text {surf }}=T_{5}$. However, the ratio model performed better than the C-parameterisation using $T_{\text {surf }}=T_{0}$, except during the first several days of the dry down period. The advantage of the ratio model is that it can estimate $T_{\mathrm{EFF}}$ using direct measurements of the soil skin temperature only, while the C-parameterisation relies on simulations to provide the necessary temperature information. 
Given the limited scope of the simulations presented here, the best-fitting values found for the parameters of the ratio model (viz., $\rho_{\min }, H_{0}$ and $P$ ) cannot be assumed to hold universally. Additional simulations are required to determine how $\rho$ varies with latitude, season, soil and vegetation properties, and meteorological conditions. The extent to which the model parameters are found to vary spatially and temporally will determine the calibration requirements for an operational application. Calibration data on soil and vegetation properties, and meteorological conditions, can only be collected with limited spatial and temporal resolution. Therefore, the effectiveness of the ratio model will ultimately depend on the stability of $\rho$ to changes in land cover and short-term (minutes to hours) weather variations.

At any particular hour between 09:00 and 17:00 hours, $\rho$ tended to decrease as the soil dried. The ratio model might thus be re-formulated to include a moisture term, but at the expense of added complexity and associated calibration requirements.

The ratio model is primarily expected to be beneficial for airborne, rather than space, operations. Airborne observations are typically conducted during daylight hours, thus presenting the need to account for diurnal variations in the temperature profile. Satellite missions, when placed in Sun synchronous orbit, will encounter the same overpass time, thus avoiding the need for an hour-of-day dependent calibration. Variations in the temperature profile will occur on a seasonal timescale, so a modified form of the ratio model may still be of benefit for space-based operations.

\section{ACKNOWLEDGEMENT}

This work was conducted with financial support from the E H Graham Centre for Agricultural Innovation and the Australian Research Council (DP0557543). The authors thank the Commonwealth Scientific and Industrial Research Organisation for providing meteorological data obtained during the National Airborne Field Experiment (2006).

\section{REFERENCES}

Choudhury, B. J., T. J. Schmugge, and T. Mo (1982). A parameterization of effective soil temperature for microwave emission. J. Geophys. Res. 87 No. C2, 1301-1304.

Das, N. N., B. P. Mohanty, M. H. Cosh, and T. J. Jackson (2008). Modeling and assimilation of root zone soil moisture using remote sensing observations in walnut gulch watershed during smex04. Remote Sens. Environ. 112, 415-429.

de Rosnay, P., J.-P. Wigneron, T. Holmes, and J.-C. Calvert (2006). Parametrisations of the effective temperature for l-band radiometry. inter-comparison and long term validation with smosrex field experiment. In C. Mätzler, P. W. Rosenkranz, A. Battaglia, and J.-P. Wigneron (Eds.), Thermal Microwave Radiation: Applications for Remote Sensing, IET Electromagnetic Waves Series 52, London, United Kingdom, pp. 312-324. The Institution of Engineering and Technology.

Flerchinger, G. N., W. P. Kustas, and M. A. Weltz (1998). Simulating surface energy fluxes and radiometric surface temperatures for two arid vegetation communities using the shaw model. J. Appl. Meteor. 37, 449-460.

Ulaby, F. T., R. K. Moore, and A. K. Fung (1986). Microwave Remote Sensing, Active and Passive. Volume III: From Theory to Applications. Norwood: Artech House.

Wang, J. R. and T. J. Schmugge (1980). An empirical model for the complex dielectric constant of soils as a function of water content. IEEE Trans. Geosci. Electron. GE-18, 288-295.

Wigneron, J.-P., A. Chanzy, P. de Rosnay, C. Rüdinger, and J.-C. Calvert (2008). Estimating the effective soil temperature at 1-band as a function of soil properties. IEEE Trans. Geosci. Remote Sens. 46, 797-807.

Wigneron, J.-P., L. Laguerre, and Y. H. Kerr (2001). A simple parameterization of the 1-band microwave emission from rough agricultural soils. IEEE Trans. Geosci. Remote Sens. 39, 1697-1707. 